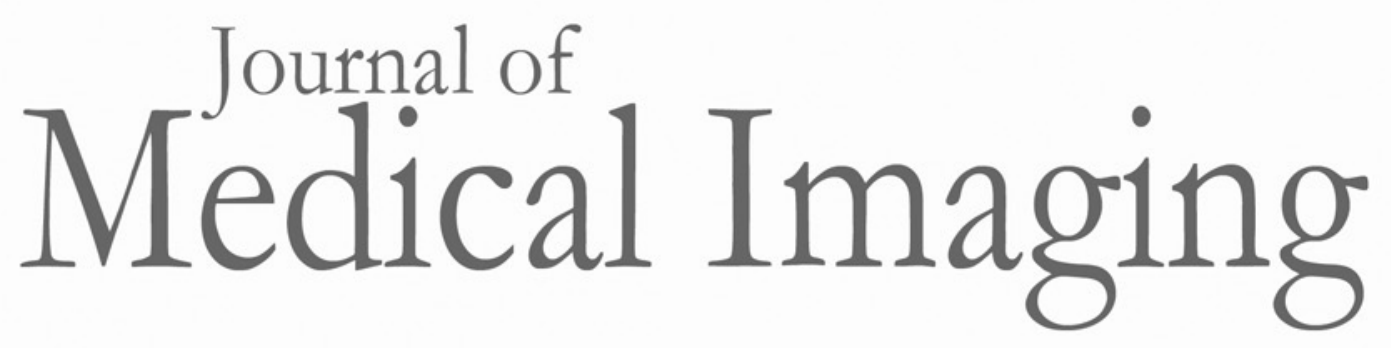

Medicallmaging.SPIEDigitalLibrary.org

\title{
JMI 2014 List of Reviewers
}

\section{SPIE.}


The Journal of Medical Imaging would like to sincerely thank the following individuals who served as reviewers in 2014 . The success of our publication hinges on the voluntary contributions of time and energy put forth by these professionals.

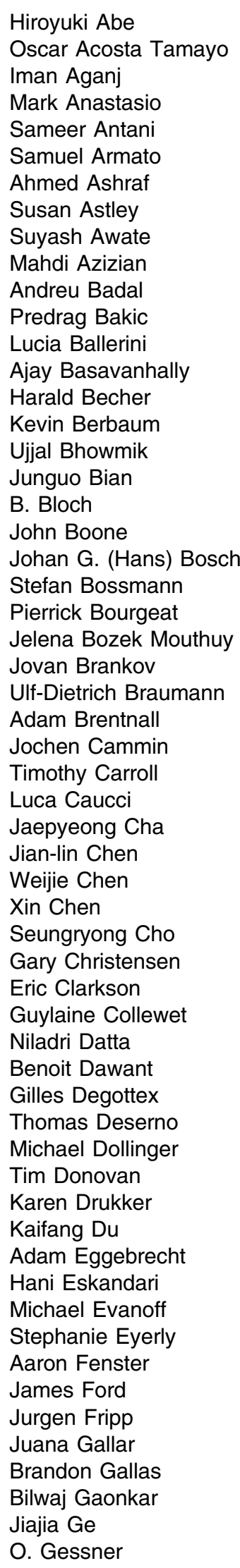

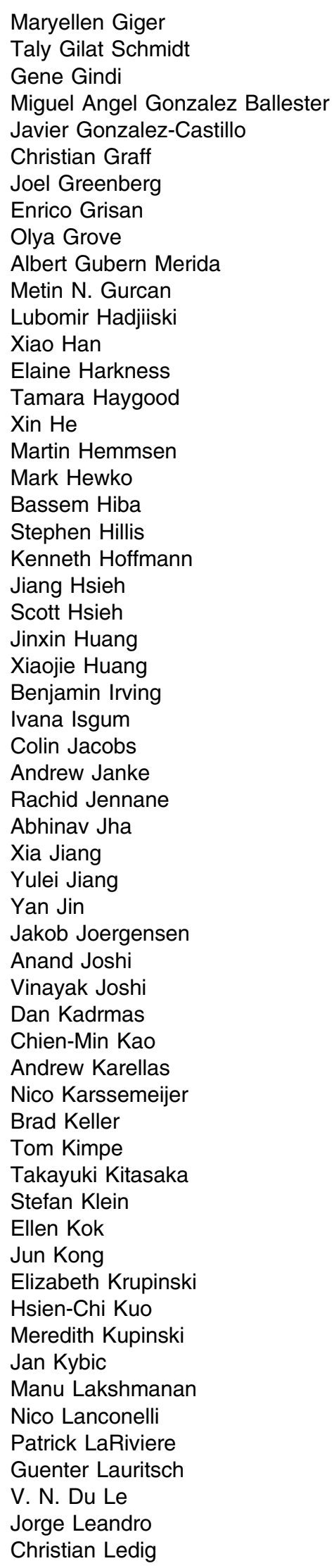

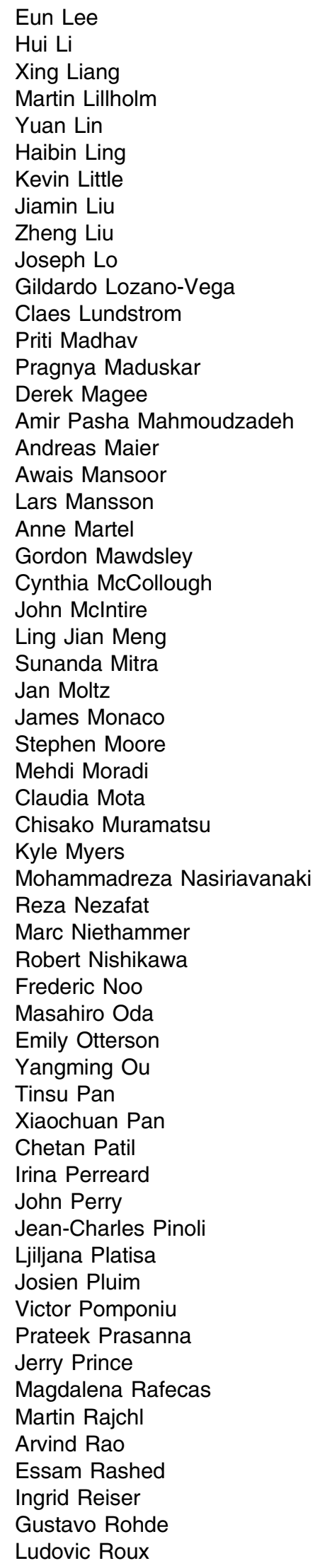


John Rowlands

Alireza Sadr

Berkman Sahiner

Frank Samuelson

Adrian Sanchez

Clarisa Sanchez

Eric Seibel

Azad Shademan

Peter Sharp

Prarthana Shrestha

Emil Sidky

Eliot Siegel

Augusto Silva

Jayanthi Sivaswamy

Yulin Song

Dima Suki

Ronald Summers

Mark Supanich

Katsuyuki Taguchi

Maxine Tan

Li Tang

Wilson Tang

Lawrence Tarbox

Jie Tian
Junichi Tokuda

Akif Burak Tosun

Georgia Tourassi

Jacques-Donald Tournier

Jens Traeger

Jayaram K. Udupa

Ruben van Engen

Anouk van Gijp

Carla van Gils

Marcel van Herk

Jifke Veenland

Marcelo Vieira

Satish Viswanath

Tao Wan

Adam Wang

Hongzhi Wang

Hui Wang

Jing Wang

Kun Wang

Zhao Wang

Weston Welge

Di Wen

Rene Werner

Miles Wernick
Bruce Whiting

Oliver Wieben

Rafael Wiemker

Bing Wu

Meng Wu

Adam Wunderlich

Hao Yang

Yuan Yao

Xin Yin

Jie Yuan

Paul Yushkevich

Federica Zanca

Zheng Zhang

Wei Zhao

Youbo Zhao

Yuanjie Zheng

Yufeng Zheng

Jiayin Zhou

Zhiheng Zhou

Lei Zhu

Quing Zhu

Reyer Zwiggelaar 